\title{
miR-203 sensitizes glioma cells to temozolomide and inhibits glioma cell invasion by targeting E2F3
}

\author{
GUODONG TANG* , JUN WU*, GELEI XIAO and LEI HUO \\ Department of Neurosurgery, Xiangya Hospital, Central South University, Changsha, Hunan 410078, P.R. China
}

Received April 10, 2014; Accepted July 28, 2014

DOI: $10.3892 / \mathrm{mmr} .2014 .3101$

\begin{abstract}
Glioma is the most common malignant and fatal primary tumor in the central nervous system in adults. Recent data has suggested a profound role for microRNAs (miRs) in cancer progression. The present study demonstrated, via quantitative polymerase chain reaction (qPCR) analysis, that miR-203 expression was markedly lower in highly invasive U87MG glioma cells and glioma tissues. Wound healing and Transwell assays demonstrated that restoration of miR-203 expression inhibited U87MG cell migration and invasion. Restoration of miR-203 expression additionally sensitized the cells to temozolomide (TMZ) as determined by MTS assay. By contrast, miR-203 inhibition in A172 cells exerted opposite effects. Bioinformatic analysis combined with experimental analysis revealed that miR-203 directly targeted E2F3 via the conserved miR-203 target site within the E2F3 3'-untranslational region. E2F3 knockdown with specific small hairpin RNA also inhibited U87MG cell migration and invasion, and sensitized them to TMZ. Importantly, miR-203 and E2F3 showed inverse expression patterns in invasive glioma tissues, as demonstrated by qPCR and luciferase assay. These results suggested that miR-203 may function as a tumor suppressor in glioma progression and that the miR-203/E2F3 axis may be a novel candidate in the development of rational therapeutic strategies for glioma.
\end{abstract}

\section{Introduction}

Glioma is the most common malignant and fatal primary tumor in the central nervous system (CNS) in adults. Though progress has been made in the development of treatments to improve patient outcomes during past decades, prognosis of glioma patients remains poor, with a median survival time of

Correspondence to: Dr Lei Huo, Department of Neurosurgery, Xiangya Hospital, Central South University, 88 Xiangya Road, Changsha, Hunan 410078, P.R. China

E-mail: huol2014@163.com

*Contributed equally

Key words: glioma, miR-203, chemosensitivity, invasion, E2F3
12-14 months. The poor prognosis of patients is mainly attributed to the high tendency of tumor invasiveness, resistance to therapy and high frequency of recurrence (1). However, the molecular mechanisms underlying these molecular events of glioma cells remain elusive.

Deregulation of microRNAs (miRNAs) has been implicated in the development and progression of almost all tumor types (2). miRNAs are single-stranded, 19-25 bp short, non-coding RNAs, and elicit their regulatory effects in post-transcriptional regulation of genes by binding to the 3'untranslated region (3'UTR) of target messenger RNA (mRNA), mainly leading to translational repression or target mRNA degradation (3). It has been estimated that miRNAs regulate up to one-third of human genes at the post-transcriptional level, suggesting that miRNAs have pivotal roles in numerous physiological and pathophysiological processes, including development, differentiation, proliferation, stress response, metabolism and apoptosis (4-7). Deregulated miRNAs may function as tumor suppressors or tumor promoters due to the diversity of miRNAs themselves (8). Emerging evidence has implicated miR-203 loss in cancer pathology, including cancer cell proliferation, invasion and drug resistance $(9,10)$. However, the function of miR-203 in glioma cells remains to be fully elucidated. The present study investigated miR-203 expression in aggressive U87MG glioma cells and glioma tissues, and assessed the effect of ectopic expression of miR-203 on invasion of U87MG cells and their sensitivity to temozolomide (TMZ) by targeting E2F3.

\section{Materials and methods}

Cell culture and tissue specimens. The human glioma cell lines A172, U87MG and U251MG, and the human embryonic kidney cell line (HEK-293T) (Cancer Research Institute of Central South University, Cambridge, MA, USA) were cultured in Dulbecco's modified Eagle's medium supplemented with $10 \%$ fetal bovine serum (HyClone, Logan City, UT, USA) at $37^{\circ} \mathrm{C}$ in a humidified atmosphere containing $5 \% \mathrm{CO}_{2}$. Fifty-two paired glioma and non-cancerous brain tissues were obtained from the Department of Neurosurgery at Xiangya Hospital of Central South University (Changsha, China) between September 2011 and March 2013. Ethical approval for human subjects was obtained from the research and ethics committee of the Xiangya Hospital of Central South University (Changsha, China) and informed consent was obtained from each patient. All tumor 
and normal tissue samples were divided into two sections and immediately snap-frozen in liquid nitrogen. Half of each sample was used for miRNA isolation and the remaining section was used for immunohistochemical (IHC) analysis.

Plasmid transfection. miR-203 expression plasmid pSilencer2.1-U6-miR-203 or empty control vector (Cancer Research Institute of Central South University) was introduced into U87MG cells with Lipofectamine 2000 (Invitrogen, Carlsbad, CA, USA) in accordance with the manufacturer's instructions in 24-well plates. Following $24 \mathrm{~h}$ of incubation, the cells were selected by incubation with $4 \mu \mathrm{g} / \mathrm{ml}$ puromycin (Sigma-Aldrich, St. Louis, MO, USA), for two weeks and the individual stable clones were analyzed by quantitative polymerase chain reaction (qPCR). U87MG cells were transfected with pRNAT-U6.1/sh-E2F3 plasmids (Genscript, Nanjing, Jiangsu, China) to knockdown E2F3 by using Lipofectamine 2000, selected with $800 \mu \mathrm{g} / \mathrm{ml} \mathrm{G418}$ and validated by western blot analysis. The miR-203 inhibitor (Qiagen, Hilden, Germany) was transfected in a $10-\mathrm{cm}$ dish using Lipofectamine 2000. Cells were harvested $48 \mathrm{~h}$ later and the subsequent experiments were performed.

miRNA-specific qPCR. miRNAs from cultured cells and tissues were isolated and purified using an miRNA isolation system (OMEGA Bio-Tek, Norcross, GA, USA). cDNA was generated with the miScript II RT Kit (Qiagen) and the qPCR was performed using the miScript SYBR Green PCR Kit (Qiagen) following the manufacturer's instructions. The miRNA sequence-specific qPCR primers for miR-203 and endogenous control RNU6 were purchased from Qiagen, and qPCR analysis was performed using the 7500 Real-Time PCR System (Applied Biosystems, Foster City, CA, USA). The gene expression threshold cycle (CT) values of miRNAs were calculated by normalizing to the internal control RNU6 and relative quantification values were calculated.

(3-(4,5-dimethylthiazol-2-yl)-5-(3-carboxymethoxyphenyl)-2(4-sulfophenyl)-2H-tetrazolium) (MTS) assay. The Cell Titer 96 ${ }^{\circledR}$ AQueous One Solution Cell Proliferation Assay kit (Promega, Madison, WI, USA) was used to determine the sensitivity of cells to TMZ. In brief, cells were seeded in 96-well plates at a density of $4 \times 10^{3}$ cells/well $(0.20 \mathrm{ml} /$ well $)$ for $24 \mathrm{~h}$ prior to use. The culture medium was replaced with fresh medium containing various concentrations of $\mathrm{TMZ}$ and incubated for $72 \mathrm{~h}$. Subsequently, MTS $(0.02 \mathrm{ml} /$ well $)$ was added to the culture medium. Following a further $2 \mathrm{~h}$ of incubation, the absorbance at $490 \mathrm{~nm}$ of each well was recorded on an ELX800 (Biotek, Winoosky, VT, USA). The growth rate was calculated as the ratio of the absorbance of the experimental well to that of the control well. The inhibition rate and the $\mathrm{IC}_{50}$ (concentration of drug resulting in $50 \%$ of control value) were also calculated.

Western blot analysis. Total proteins were extracted from corresponding cells using the RIPA buffer (Pierce, Rockford, IL, USA) in the presence of Protease Inhibitor Cocktail (Pierce). The protein concentration of the lysates was measured using a BCA Protein Assay Kit (Pierce), loaded and separated by $10 \%$ SDS-PAGE and then transferred to a polyvinylidene fluoride membrane (Millipore, Billerica, MA, USA). The primary antibodies used for analysis included mouse anti-E2F3 monoclonal antibody (1:800) and mouse anti- $\beta$-actin monoclonal antibody (1:2,000; Santa Cruz, Dallas, TX, USA).

Luciferase reporter assay. Two single strands of the wild-type 3'UTR with miR-203 binding site and two single strands of the mutant type with seven bases deleted in the miR-203 binding site (as mutant control), of E2F3 were synthesized with restriction sites for SpeI and HindIII located at both ends of the oligonucleotides for further cloning. The single strand DNA sequences were: Wild-type 3'UTR of E2F3 sense, 5'-CTAGTCAGCAATCTTCCTTAATAGCATTTCAAGCCG TGCCTTCTCCCGCAGAATGCA-3' and antisense, 5'-AGCTTGCATTCTGCGGAGAAGGCACGGCTTGAAAT GCTATTAAGGAAGATTGCTGA-3'; and the mutant type 3'UTR of E2F3 sense, 5'-CTAGTCAGCAATCTTCCTT AATAG-------AGCCGTGCCTTCTCCGCAGAATGCA-3' and antisense, 5'-AGCTTGCATTCTGCGGAGAAG GCACGGCT------CTATTAAGGAAGATTGCTGA-3'. The corresponding sense and antisense strands were annealed and subsequently cloned into pMir-Report plasmids downstream of the firefly luciferase reporter gene. Cells were seeded in 96-well plates and co-transfected with pMir-Report luciferase vector, pRL-TK Renilla luciferase vector and miR-203 expression vector (Cancer Research Institute of Central South University). Following $48 \mathrm{~h}$ of incubation, luciferase activity was determined using a Dual-Luciferase Reporter Assay System (Promega Corp., Madison, WI, USA) where Renilla luciferase activity was used as an internal control and the firefly luciferase activity was calculated as the mean \pm standard deviation (SD) after being normalized against Renilla luciferase activity.

Wound healing assay. Cells were synchronized to ensure a homogeneous and viable cell monolayer prior to wounding. Equal numbers of cells were seeded into six-well culture plates. When cell confluence reached $\sim 90 \%$ at $48 \mathrm{~h}$ post-transfection, an artificial homogenous wound was created on the monolayer with a sterile plastic $100-\mu l$ micropipette tip. Following wounding, debris was removed by washing the cells with serum-free medium. Migration of cells into the wound was observed after $36 \mathrm{~h}$. Images were captured of cells which migrated into the wounded area or cells with extended protrusions from the border of the wound. Related observation was done under the contrast phase model on microscope from Leica (Wetzlar, Germany).

Transwell cell invasion assay. Cells were detached and resuspended in serum-free medium. Cells $\left(1 \times 10^{5}\right.$ cells/well) were then plated into Matrigel ${ }^{\circledR}$-coated invasion chambers (Becton Dickinson, Franklin Lakes, NJ, USA) and allowed to invade for $24 \mathrm{~h}$. The remaining cells in the chambers were removed by cotton swabs and the invading cells on the lower surface of the chambers were fixed with $70 \%$ ethanol and then stained with hematoxylin (Sigma-Aldrich). The number of invading cells was calculated by counting three different fields under a phase contrast microscope (Leica Wetzlar, Germany).

IHC analysis. Formalin-fixed, paraffin-embedded tissue specimens were cut into $4-\mu \mathrm{m}$ sections. The specimens were deparaffinized in xylene (Sigma-Aldrich) and rehydrated using 

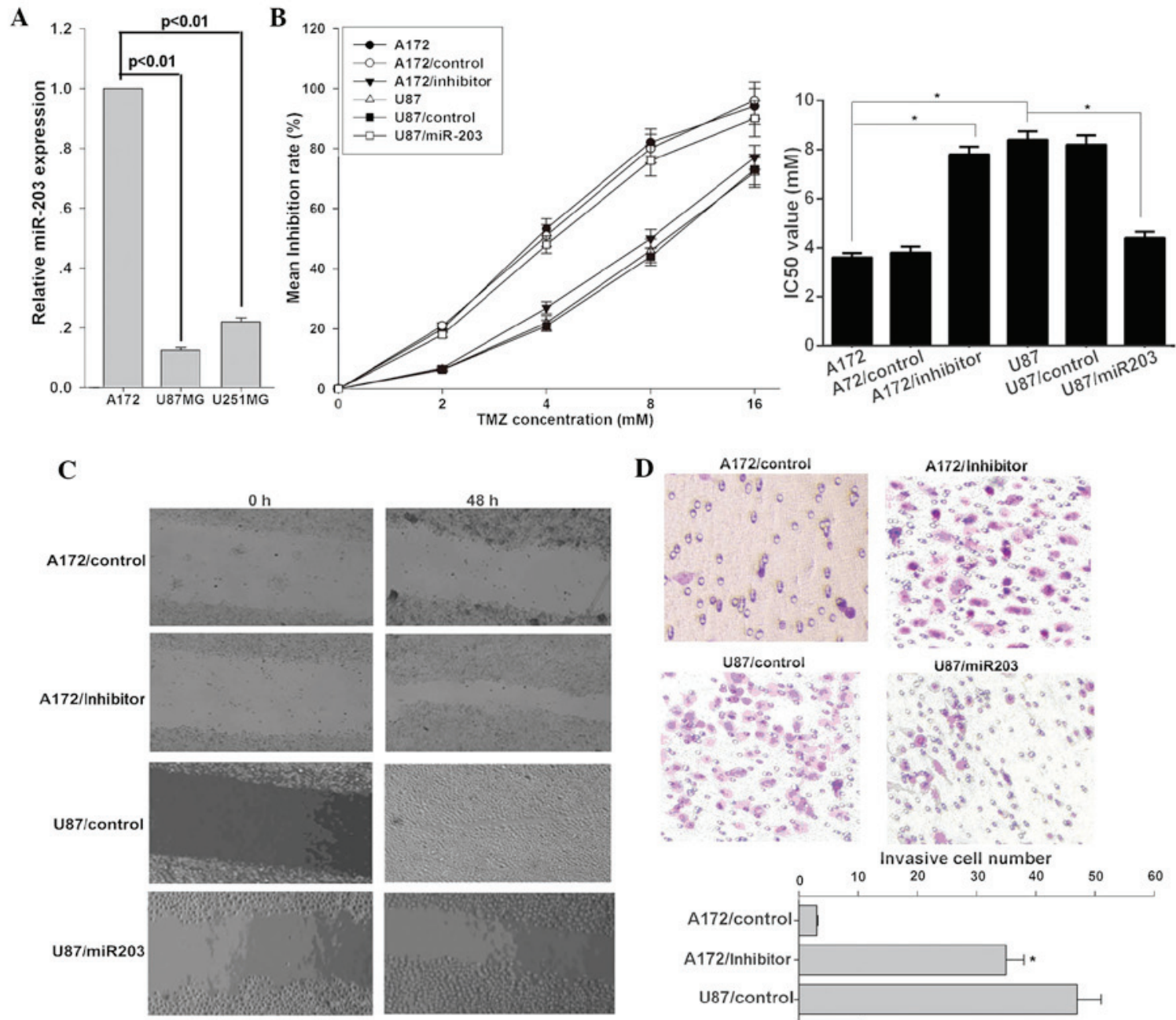

E
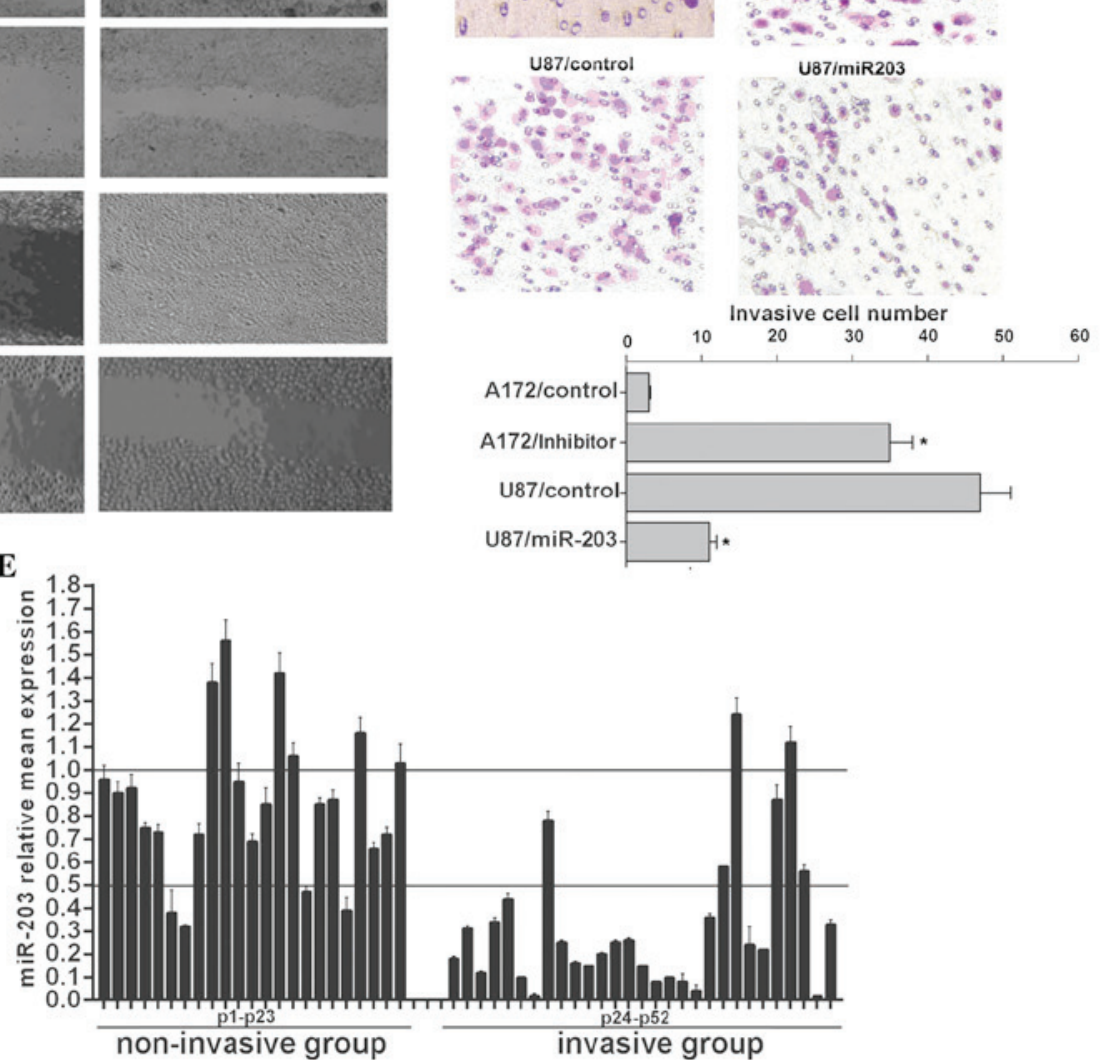

Figure 1. miR-203 sensitizes glioma cells to TMZ and inhibits their migration and invasion. (A) The expression of miR-203 in glioma cell lines with different invasive potential was tested by quantiative polymerase chain reaction. (B) Dose-inhibition rate curves plotted from the MTS assay results collected from three independent experiments. The effect of miR-203 interference on glioma cell line response to TMZ (right) and the IC $\mathrm{C}_{50}$ values of $\mathrm{TMZ}$, $\mathrm{P}<0.01$. (C) Wound healing assay demonstrated that miR-203 inhibited glioma cell migration (magnification, x10). (D) Transwell cell invasion assay was performed on control cell lines and cell lines subjected to miR-203 expression interference (magnification, $\mathrm{x} 20$ ). vs respective control ${ }^{*} \mathrm{P}<0.01$. (E) Expression pattern of miR-03 in non-invasive and invasive human glioma tissues. TMZ, temozolomide; miR, microRNA; MTS, (3-(4,5-dimethylthiazol-2-yl)-5-(3-carboxymethox yphenyl)-2-(4-sulfophenyl)-2H-tetrazolium).

a series of graded alcohols following being dried at $62^{\circ} \mathrm{C}$ for two hours. The tissue slides were then treated with $3 \%$ hydrogen peroxide (Sigma-Aldrich) in methanol for $15 \mathrm{~min}$. To exhaust endogenous peroxidase activity, the antigens were retrieved in $0.01 \mathrm{M}$ sodium citrate buffer (pH 6.0) (Sigma-Aldrich) using a microwave oven. Following one hour of preincubation in
$10 \%$ goat serum, the specimens were incubated with primary antibody (1:50; Santa Cruz) at $4^{\circ} \mathrm{C}$ overnight. The tissue slides were treated with a non-biotin horseradish peroxidase detection system according to the manufacturer's instructions (DAKO, Carpinteria, CA, USA). Two pathologists who specialize in liver cancer evaluated the results of IHC. 

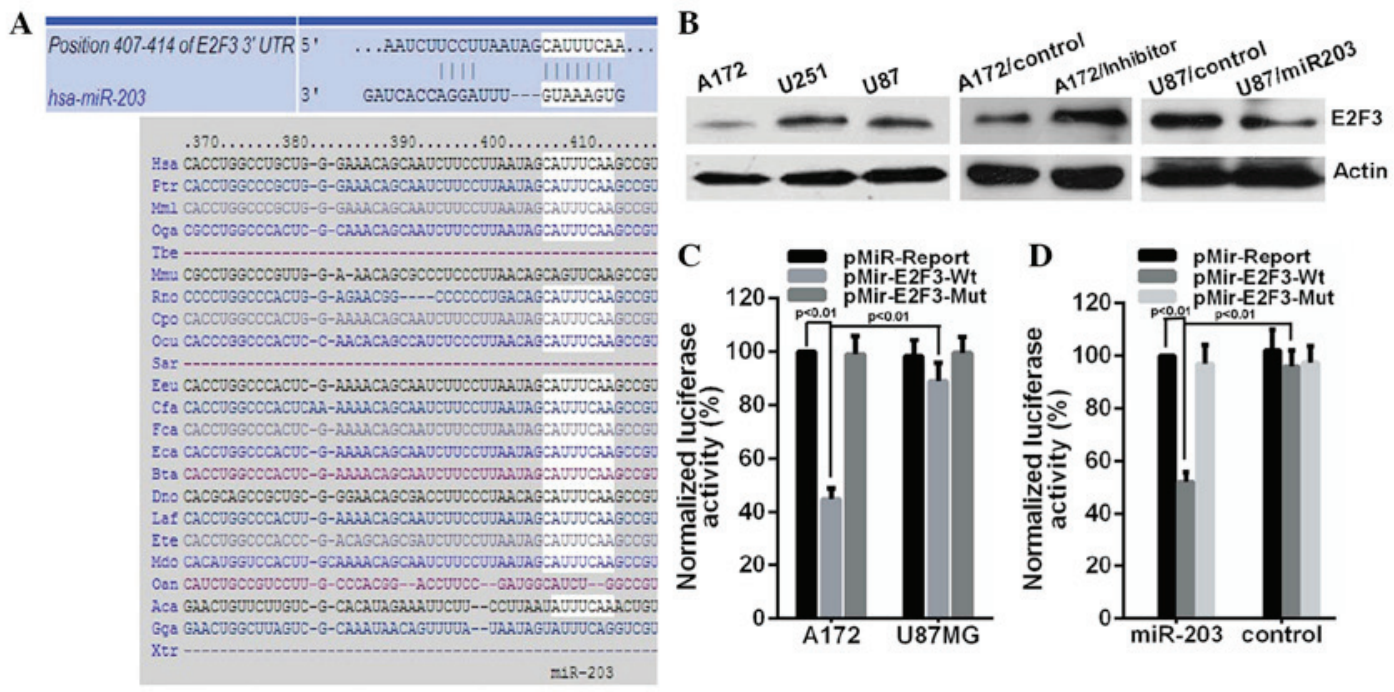

Figure 2. miR-203 targets E2F3 in glioma cells. (A) Schematic of the predicted miR-203 target site in the human E2F3 3'UTR, which is broadly conserved among vertebrates. (B) Inverse relationship between miR-203 and E2F3 protein levels are shown in selected glioma cell lines. (C and D) miR-203 suppressed the activity of the luciferase gene linked to the 3'UTR of E2F3, and a Renilla luciferase reporter was used for normalization. The data were obtained from three independent experiments. The mean of the results from A172 transfected with the pMir-control and from human embryonic kidney 293T cells transfected with the pMir-control and miR-203 were set as 100\%. 3'UTR, 3'-untranslational region; miR, microRNA; Wt, wild-type; Mut, mutant; hsa, Homo sapiens.

Statistical analysis. Each experiment was repeated at least three times. Statistical analysis was performed using SPSS 16.0 (International Business Machines, Armonk, NY,USA). Student's t-test was used to analyze the statistical difference. Results were presented as the mean $\pm \mathrm{SD}$. $\mathrm{P}<0.05$ was considered to indicate a statistically significant difference between values.

\section{Results}

miR-203 sensitizes glioma cells to TMZ and inhibits their invasion. To determine the role of miR-203 in glioma, the miR-203 expression pattern in selected glioma cell lines with varying aggressive potential was examined. miR-203 was markedly decreased in U87MG and U251MG cell lines with highly invasive potential compared to that in the less invasive A172 cells (Fig. 1A). In order to verify the correlation between the biological characteristics of glioma cells and miR-203 expression, a series of assays were employed. The present study demonstrated that U87MG cells were more resistant to TMZ (Fig. 1B) and more invasive (Fig. 1C and D) than A172 cells. Ectopic miR-203 expression significantly sensitized U87MG cells to TMZ and inhibited U87MG cell migration and invasion (Fig. 1C and D). A wound healing assay demonstrated that miR-203-transduced U87MG cells exhibited markedly slower migration compared with vector-control cells (Fig. 1C). Through the cell invasion assay, the effect of miR-203 on cell invasion, a key determinant of malignant progression and metastasis, was assessed. As demonstrated in Fig. 1D, miR-203 led to significantly decreased invasion (miR-203 group, $11 \pm 1$ cells per field; control group, $47 \pm 4$ cells per field) of U87MG cells. Inversely, the miR-203 inhibitor attenuated the sensitivity of A172 cells to TMZ (Fig. 1B) and promoted migration (Fig. 1C) and invasion (miR-203 inhibitor group, $35 \pm 3$ cells per field; control group, $3 \pm 0.2$ cells per field) (Fig. 1D). To investigate the association between miR-203 and glioma invasion, a total of 52 clinical glioma tissue samples and paired non-cancerous brain samples were collected and divided into non-invasive (23 samples) and invasive groups (29 samples). The present study demonstrated that miR-203 was significantly downregulated in the invasive group with $\sim 79.31 \%$ of the cases displaying $>2$-fold downregulation. However, in the non-invasive group only $\sim 13.04 \%$ of the cases displayed $>2$-fold downregulation $(\mathrm{P}<0.01)$ (Fig. 1E). These results revealed a functional role for miR-203 in cell invasion and chemosensitivity in glioma cells, thus suggesting a mechanism by which downregulation of miR-203 may contribute to tumor progression and chemotherapy resistance in glioma.

miR-203 directly targets E2F3 in glioma cells. To analyze the molecular mechanisms of miR-203 involvement in glioma cells, its target gene was sought. The public database TargetScan (http://www.targetscan.org) was used to predict potential targets of miR-203. E2F3, with critically conserved binding sites, was selected for further characterization (Fig. 2A). To validate the potential targeting of E2F3 by miR-203, protein expression of E2F3 in glioma cells that had been subjected to miR-203 expression interference was examined. The present study demonstrated that E2F3 protein expression in U87MG and U251MG cells was markedly greater than that in A172 cells (Fig. 2B). E2F3 in U87MG cells was significantly decreased following ectopic miR-203 expression. However, miR-203 inhibitor upregulated E2F3 protein level in A172 cells. To assess whether E2F3 is a direct target of miR-203, a luciferase reporter vector containing the putative E2F3 3'UTR target site for miR-203 downstream of the luciferase gene (pMir-E2F3-Wt) and a mutant version containing a deletion of $7 \mathrm{bp}$ in the seed region was constructed (pMir-E2F3-Mut). As demonstrated in Fig. 2C, the luciferase activity of pMir-E2F3-Wt in U87MG was 44.78\% higher than that of the A172 cells, while luciferase activity of pMir-E2F3-Mut was unaffected, suggesting that endogenous 

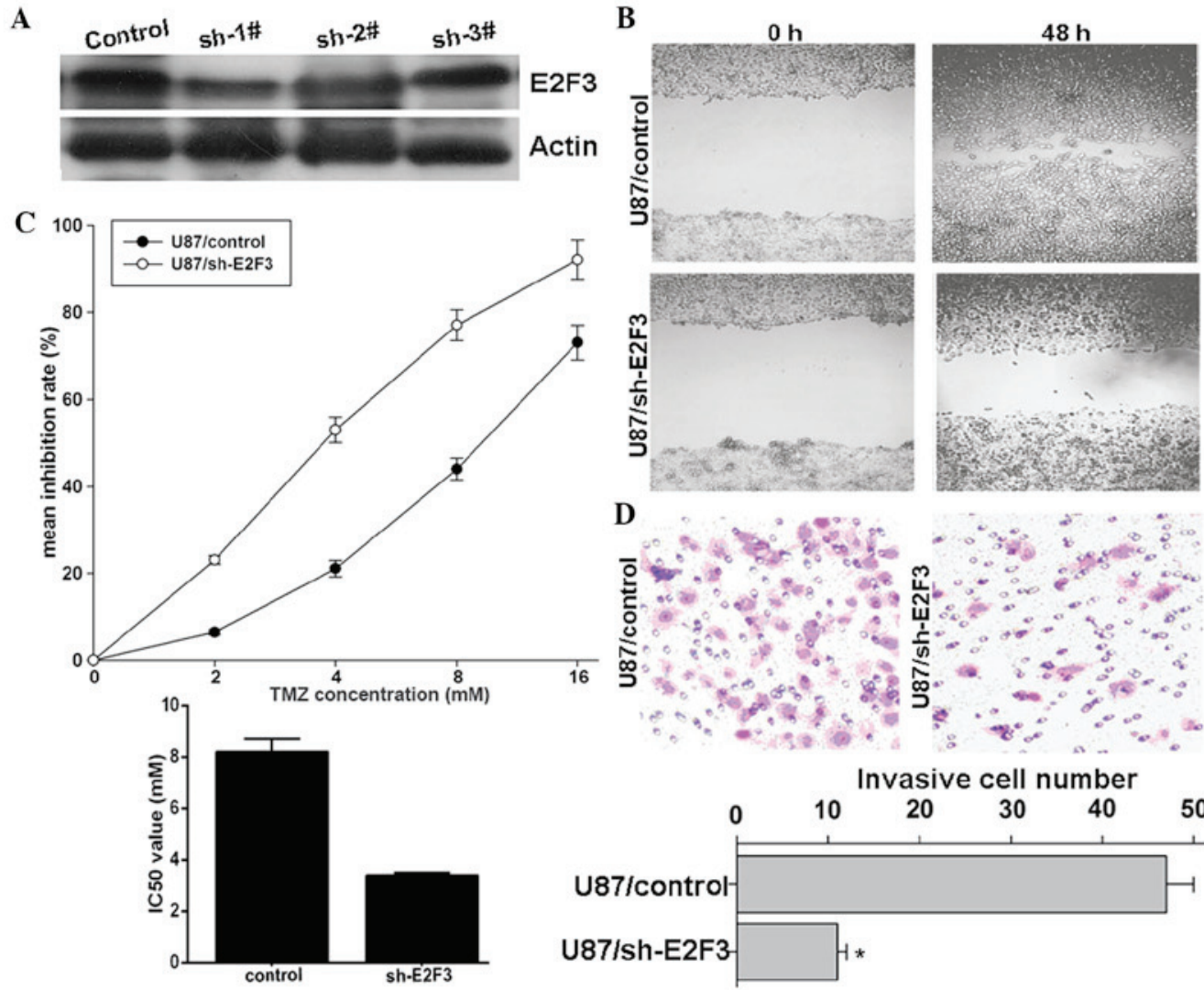

E
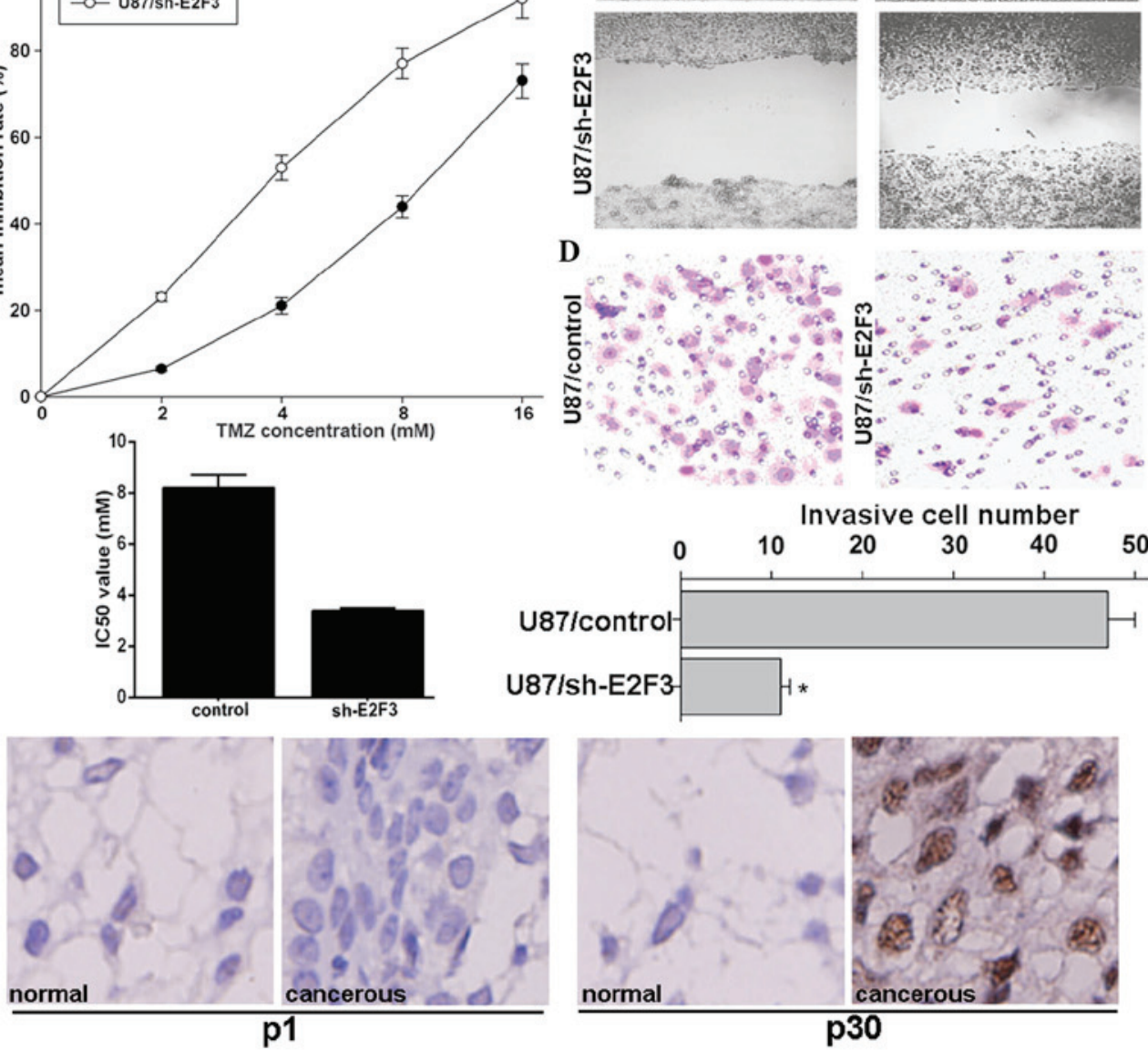

\section{.}

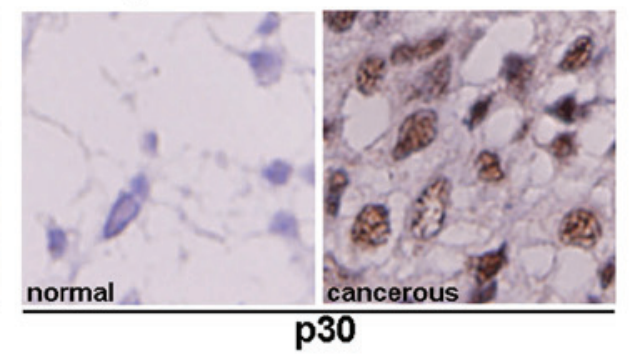

Figure 3. E2F3 knockdown sensitizes U87MG cells to TMZ and inhibits their invasion. (A) E2F3 expression change mediated by sh-E2F3 demonstrated that sh-1\# significantly reduced E2F3 protein expression. (B) Wound healing assay demonstrated that E2F3 knockdown inhibited U87MG cell migration (magnification, x10). (C) Dose-inhibition rate curves were plotted from the MTS assay results collected from three independent experiments. These demonsrated that sh-E2F3 significantly decreased cell viability upon TMZ treatment, with decreased $\mathrm{IC}_{50}$ values. (D) Transwell cell invasion assay was performed on the control cell line and E2F3 knockdown cell line (magnification, $\mathrm{x} 20$ ). "P<0.01 vs control. (E) Immunohistochemical assay of E2F3 expression in glioma tissues and paired normal tissues. The left panel represents paired glioma and normal tissues from the non-invasive group and the right panel represents paired glioma and normal tissues from the invasive group (magnification, x20). TMZ, temozolomide; sh-E2F3, short hairpin E2F3; MTS, (3-(4,5-dimethylthiazol-2-yl)-5-(3-carboxymethoxyp henyl)-2-(4-sulfophenyl)-2H-tetrazolium); p, patient.

miR-203 suppressed gene expression with the seed region in the 3'UTR of miRNA. Moreover, the luciferase reporter assay performed on HEK293T cells revealed that miR-203 reduced the luciferase activity of the vector with the wild-type E2F3 3'UTR by $\sim 52.27 \%$. However, the mutant version abrogated the repressive ability of miR-203 (Fig. 2D). These results demonstrated the specificity of miR-203 targeting of E2F3.

E2F3 knockdown enhances the effect of TMZ and inhibits invasion of glioma cells. To investigate whether miR-203 enhances chemotherapeutic sensitivity and inhibits invasion through inhibition of E2F3 expression, E2F3 was knocked down using pRNAT-U6.1/sh-E2F3 plasmid expressing a short hairpin RNA (shRNA) targeting E2F3. Compared with the control, sh-1\# markedly decreased E2F3 protein expression (Fig. 3A), and therefore, was selected for further study. The functional effect of E2F3 knockdown on glioma cells was then assessed. The preset study revealed that E2F3 knockdown significantly inhibited U87MG-cell migration as demonstrated by the wound-healing assay (Fig. 3B), enhanced sensitivity of U87MG cells to TMZ as demonstrated by the MTS assay (Fig. 3C) and inhibited invasion as demonstrated by the Transwell assay (Fig. 3D), similarly to the effect of ectopic miR-203 overexpression. Importantly, in the selected 52 clinical glioma tissues, high E2F3 protein expression was detected in the invasive group, compared to that of the non-invasive group. Representative data were obtained from patient one with high miR-203 expression and patient 30 with low miR-203 expression, respectively (Fig. 3E). These results suggested that E2F3 may be involved in the mechanism of miR-203 in invasion inhibition in glioma cells. 


\section{Discussion}

Although the roles of miRNAs in cancer have been widely studied via analysis of specific genes targeted and their biological functions as well as their significance in cancer development, progression and therapeutic response, their contribution to cancer remains to be fully elucidated (8). For example, knowledge concerning the role of miR-203 in cancer, particularly in human glioma, remains incomplete. Recently, miR-203 was found to be frequently downregulated in cervical cancer tumors and cell lines due to methylation of the miR-203 promoter. miR-203 was also capable of suppressing cervical cancer cell proliferation, tumor growth, and angiogenesis by directly targeting vascular endothelial growth factor A (11). Zhang et al (12) observed that hypermethylated miR-203 was involved in Nickel-induced tumorigenesis and that miR-203 may suppress tumorigenesis, at least in part, by targeting Abelson murine leukemia viral oncongene homolog 1 . Another study revealed that miR-203 was significantly downregulated in esophageal cancer and overexpression of miR-203 in esophageal cancer cells markedly increased cell apoptosis and inhibited cell proliferation, migration and invasion as well as tumor growth (13). The present study, for the first time, to the best of our knowledge, demonstrated that miR-203 expression was markedly lower in the more aggressive U87MG glioma cells than that in A172 glioma cells. Ectopic expression of miR-203 significantly inhibited the invasion of U87MG cells and sensitized them to TMZ. Inversely, an miR-203 inhibitor markedly promoted the invasion of A172 cells and attenuated their sensitivity to TMZ. Moreover, miR-203 expression was negatively associated with the invasive potential of glioma cells. In regard to the mechanism of miR-203 in glioma, bioinformatic analysis combined with a luciferase reporter assay indicated that miR-203 may directly target E2F3. As expected, E2F3 knockdown demonstrated the same effects as ectopic expression of miR-203 on U87MG cells.

E2F3 belongs to the E2F transcription factor family, which is well conserved and widely known for its role in proliferation and cell-cycle progression (14). Genetic mouse models provided the first evidence demonstrating that E2F3 largely contributed to retinoblastoma protein-attributed tumorigenic events, such as the reduction of retinoblastoma-deficient pituitary adenomas in E2F3-deficient mice (15), and additionally inhibited the development of preneoplastic lesions of the lung (16). Furthermore, the loss of E2F3, in combination with E2F1 and E2F2, reduced hyperplasia in intestinal epithelia (17). In human patients, there is mounting evidence that deregulation of E2F3 protein contributes to tumor formation. The E2F3 gene, located on chromosome 6p22, was found to be amplified in a subset of retinoblastoma, breast, and urinary bladder carcinomas (18-23). In bladder cancer, E2F3 amplification is associated with more malignant and invasive cancers $(20,21)$. E2F3 overexpression has also been reported in lung, ovarian and prostate cancer. Importantly, E2F3 levels independently predict clinical outcome in patients with prostate cancer (24-26). Thus, E2F3 is closely associated with cancer development and progression. In previous studies, the overexpression of E2F3 has been mainly attributed to gene amplification. Recently, studies have focussed greater attention on the study of the epigenetic mechanisms underlying gene expression regulation (9). A study by Feng et al (27) revealed that miR-200b was capable of targeting E2F3 to reverse the chemoresistance of docetaxel-resistant human lung adenocarcinoma cells. In the present study, for the first time, to the best of our knowledge, it was demonstrated that the role of E2F3 in glioma cells was associated with the response to chemotherapy and invasion. The mechanisms responsible for the downregulation of miR-203 and the role of E2F3 in glioma will be explored in a forthcoming study.

In conclusion, the present study indicated that miR-203 was reversely associated with migration and invasion, and positively associated with chemosensitivity in glioma cells. E2F3 was shown to be a novel target of miR-203 and E2F3 knockdown exerted a similar effect to that of miR-203 overexpression. These results indicate that miR-203 may act as a tumor suppressor by targeting E2F3 in glioma cells and that miR-203/E2F3 may be a novel candidate for developing rational therapeutic strategies in glioma treatment.

\section{Acknowledgements}

The study was supported by the project of Science and Technology of Hunan Province, China (no. 2010SK3103).

\section{References}

1. Wen PY and Kesari S: Malignant gliomas in adults. N Engl J Med 359: 492-507, 2008.

2. Hammond SM: MicroRNAs as oncogenes. Curr Opin Genet Dev 16: 4-9, 2006.

3. Croce CM and Calin GA: miRNAs, cancer and stem cell division. Cell 122: 6-7, 2005.

4. Ambros V: MicroRNA pathways in flies and worms: growth, death, fat, stress and timing. Cell 113: 673-676, 2003.

5. Brennecke J, Hipfner DR, Stark A, Russell RB and Cohen SM: Bantam encodes a developmentally regulated microRNA that controls cell proliferation and regulates the proapoptotic gene hid in Drosophila. Cell 113: 25-36, 2003.

6. Chen CZ, Li L, Lodish HF and Bartel DP: MicroRNAs modulate hematopoietic lineage differentiation. Science 303: 83-86, 2004.

7. Cheng AM, Byrom MW, Shelton J and Ford LP: Antisense inhibition of human miRNAs and indications for an involvement of miRNA in cell growth and apoptosis. Nucleic Acids Res 33: 1290-1297, 2005.

8. Iorio MV and Croce CM: MicroRNAs in cancer: small molecules with a huge impact. J Clin Oncol 27: 5848-5856, 2009.

9. Zhang Z, Zhang B, Li W, Fu L, Fu L, Zhu Z and Dong JT: Epigenetic silencing of miR-203 upregulates SNAI2 and contributes to the invasiveness of malignant breast cancer cells. Genes Cancer 2: 782-791, 2011.

10. Li Y, Yuan Y, Tao K, Wang X, Xiao Q, Huang Z, Zhong L, Cao W, Wen $\mathrm{J}$ and Feng W: Inhibition of BCR/ABL protein expression by miR-203 sensitizes for imatinib mesylate. PloS One 8: e61858, 2013.

11. Zhu X, Er K, Mao C, Yan Q, Xu H, Zhang Y,Zhu J, Cui F, Zhao W and Shi H: miR-203 suppresses tumor growth and angiogenesis by targeting VEGFA in cervical cancer. Cell Physiol Biochem 2: 64-73, 2013.

12. Zhang J, Zhou Y, Wu YJ, Li MJ, Wang RJ, Huang SQ, Gao RR, Ma L, Shi HJ and Zhang J: Hyper-methylated miR-203 dysregulates ABL1 and contributes to the nickel-induced tumorigenesis. Toxicol Lett 223: 42-51, 2013.

13. Zhang F, Yang Z, Cao M, Xu Y, Li J, Chen X, Gao Z, Xin J, Zhou S, Zhou Z, et al: MiR-203 suppresses tumor growth and invasion and down-regulates MiR-21 expression through repressing Ran in esophageal cancer. Cancer Lett 342: 121-129, 2014.

14. van den Heuvel S and Dyson NJ: Conserved functions of the pRB and E2F families. Nat Rev Mol Cell Biol 9: 713-724, 2008. 
15. Ziebold U, Lee EY, Bronson RT and Lees JA: E2F3 loss has opposing effects on different pRB-deficient tumors, resulting in suppression of pituitary tumors but metastasis of medullary thyroid carcinomas. Mol Cell Biol 23: 6542-6552, 2003.

16. Parisi T, Yuan TL, Faust AM, Caron AM, Bronson R and Lees JA: Selective requirements for E2f3 in the development and tumorigenicity of $\mathrm{Rb}$-deficient chimeric tissues. Mol Cell Biol 27: 2283-2293, 2007.

17. Chong JL, Wenzel PL, Sáenz-Robles MT, Nair V, Ferrey A, Hagan JP, Gomez YM, Sharma N, Chen HZ, Ouseph M, et al: E2f1-3 switch from activators in progenitor cells to repressors in differentiating cells. Nature 462: 930-934, 2009.

18. Veltman JA, Fridlyand J, Pejavar S, Olshen AB, Korkola JE, DeVries S, Carroll P, Kuo WL, Pinkel D, Albertson D, et al: Array-based comparative genomic hybridization for genome-wide screening of DNA copy number in bladder tumors. Cancer Res 63: 2872-2880, 2003.

19. Orlic M, Spencer CE, Wang L and Gallie BL: Expression analysis of 6 p22 genomic gain in retinoblastoma. Genes Chromosomes Cancer 45: 72-82, 2006

20. Oeggerli M, Tomovska S, Schraml P, Calvano-Forte D, Schafroth S, Simon R, Gasser T, Mihatsch MJ and Sauter G: E2F3 amplification and overexpression is associated with invasive tumor growth and rapid tumor cell proliferation in urinary bladder cancer. Oncogene 23: 5616-5623, 2004

21. Feber A, Clark J, Goodwin G, Dodson AR, Smith PH, Fletcher A, Edwards S, Flohr P, Falconer A, Roe T, et al: Amplification and overexpression of E2F3 in human bladder cancer. Oncogene 23 $1627-1630,2004$
22. Grasemann C, Gratias S, Stephan H, Schüler A, Schramm A, Klein-Hitpass L, Rieder H, Schneider S, Kappes F, Eggert A and Lohmann DR: Gains and overexpression identify DEK and E2F3 as targets of chromosome 6p gains in retinoblastoma. Oncogene 24: 6441-6449, 2005.

23. Andre F, Job B, Dessen P, Tordai A, Michiels S, Liedtke C, Richon C, Yan K, Wang B, Vassal G, et al: Molecular characterization of breast cancer with high-resolution oligonucleotide comparative genomic hybridization array. Clin Cancer Res 15: 441-451, 2009.

24. Foster CS, Falconer A, Dodson AR, Norman AR, Dennis N, Fletcher A, Southgate C, Dowe A, Dearnaley D, Jhavar S, et al: Transcription factor E2F3 overexpressed in prostate cancer independently predicts clinical outcome. Oncogene 23: 5871-5879, 2004.

25. Borczuk A, Gorenstein L, Walter KL, Assaad AA, Wang L and Powell CA: Non-small-cell lung cancer molecular signatures recapitulate lung developmental pathways. Am J Pathol 163: 1949-1960, 2003.

26. Lu KH, Patterson AP, Wang L, Marquez RT, Atkinson EN, Baggerly KA, Ramoth LR, Rosen DG, Liu J, Hellstrom I, et al: Selection of potential markers for epithelial ovarian cancer with gene expression arrays and recursive descent partition analysis. Clin Cancer Res 10: 3291-3300, 2004.

27. Feng B, Wang R, Song HZ and Chen LB: MicroRNA-200b reverses chemoresistance of docetaxel-resistant human lung adenocarcinoma cells by targeting E2F3. Cancer 118: 3365-3376, 2012 . 